\title{
Photoluminescence studies of erbium-doped GaAs under hydrostatic pressure
}

\author{
T. D. Culp, U. Hömmerich, ${ }^{\text {a) }}$ J. M. Redwing, ${ }^{\text {b) }}$ T. F. Kuech, and K. L. Bray ${ }^{\mathrm{c})}$ \\ Department of Chemical Engineering, University of Wisconsin, 1415 Engineering Drive, Madison, \\ Wisconsin 53706
}

(Received 27 December 1996; accepted for publication 14 March 1997)

The photoluminescence properties of metal-organic chemical vapor deposition GaAs:Er were investigated as a function of temperature and applied hydrostatic pressure. The ${ }^{4} I_{13 / 2} \rightarrow{ }^{4} I_{15 / 2} \mathrm{Er}^{3+}$ emission energy was largely independent of pressures up to $56 \mathrm{kbar}$ and temperatures between 12 and $300 \mathrm{~K}$. Furthermore, no significant change in the low temperature emission intensity was observed at pressures up to and beyond the $\Gamma-X$ crossover at $\sim 41 \mathrm{kbar}$. In contrast, $\mathrm{Al}_{x} \mathrm{Ga}_{1-x}$ As:Er alloying studies have shown a strong increase in intensity near the $\Gamma-X$ crossover at $x \sim 0.4$. These results suggest that the enhancement is most likely due to a chemical effect related to the presence of $\mathrm{Al}$, such as residual oxygen incorporation, rather than a band structure effect related to the indirect band gap or larger band gap energy. Modeling the temperature dependence of the $1.54 \mu \mathrm{m} \mathrm{Er}{ }^{3+}$ emission intensity and lifetime at ambient pressure suggested two dominant quenching mechanisms. At temperatures below approximately $150 \mathrm{~K}$, thermal quenching is dominated by a $\sim 13 \mathrm{meV}$ activation energy process which prevents $\mathrm{Er}^{3+}$ excitation, reducing the intensity, but does not affect the $\mathrm{Er}^{3+}$ ion once it is excited, leaving the lifetime unchanged. At higher temperatures, thermal quenching is governed by a $\sim 115 \mathrm{meV}$ activation energy process which deactivates the excited $\mathrm{Er}^{3+}$ ion, quenching both the intensity and lifetime. At $42 \mathrm{kbar}$, the low activation energy process was largely unaffected, whereas the higher activation energy process was significantly reduced. These processes are proposed to be thermal dissociation of the Er-bound exciton, and energy back transfer, respectively. A model is presented in which the Er-related electron trap shifts up in energy at higher pressure, increasing the activation energy to back transfer, but not affecting thermal dissociation of the bound exciton through hole emission. (C) 1997 American Institute of Physics. [S0021-8979(97)03912-1]

\section{INTRODUCTION}

In the past several years, there has been a strong interest in rare-earth doped semiconductors because of their potential to combine sharp rare-earth luminescence with the convenience of electrical excitation via the semiconductor host. ${ }^{1,2}$ One desirable feature of rare-earth luminescence is that the emission wavelength is largely independent of both the host material and temperature, because the filled outer $5 s$ and 5 $p$ electron shells screen transitions within the inner $4 f$ electron shell from interaction with the host. $\mathrm{Er}^{3+}$ doped materials have been of particular interest because the ${ }^{4} I_{13 / 2} \rightarrow{ }^{4} I_{15 / 2}$ emission at $1.54 \mu \mathrm{m}$ matches the minimum loss region of silica fibers used in optical communications.

Development of practical devices based on rare-earth doped semiconductors has been hindered by low room temperature efficiencies. In order to improve the efficiency, we need a better understanding of the nature of rare-earth centers in the semiconductor lattice, the relationship of rareearth related levels to the semiconductor band structure, and the basic mechanisms of rare-earth excitation and thermal quenching. Several recent studies have sought to address these needs. ${ }^{1,2}$

\footnotetext{
${ }^{a)}$ Current address: Research Center for Optical Physics, Hampton University, Hampton, VA 23668.

${ }^{b}$ Current address: Advanced Technology Materials, Danbury, CT 06810.

${ }^{c}$ Corresponding author. Electronic mail: bray@engr.wisc.edu
}

In this article, we examined the luminescence properties of GaAs:Er as a function of temperature and applied hydrostatic pressure. High pressure is a useful tool for studying the properties of impurity levels in semiconductors because of its ability to influence impurity states and the host band structure. Pressure is also capable of altering thermal energy barriers and can be used to elucidate excitation and quenching mechanisms. In many respects, pressure studies provide information similar to alloying studies. However, a key advantage to high pressure is the ability to influence energy levels and energy barriers in the absence of complicating chemical effects that often accompany alloying.

\section{EXPERIMENT}

Epitaxial GaAs films were prepared by metal-organic chemical vapor deposition (MOCVD) in a horizontal, lowpressure (78 Torr) reactor using $\left(\mathrm{CH}_{3}\right)_{3} \mathrm{Ga}$ and $\mathrm{AsH}_{3}$. Palladium diffused $\mathrm{H}_{2}$ was used as the carrier gas. Erbium doping was accomplished with a cyclopentadienyl $(\mathrm{Cp})$ based precursor, $(t \text {-butylCp })_{3}$ Er. The layers were deposited on semiinsulating (100) oriented GaAs substrates at $650^{\circ} \mathrm{C}$. The $\mathrm{V} / \mathrm{III}$ ratio $\left[\mathrm{AsH}_{3} /\left(\mathrm{CH}_{3}\right)_{3} \mathrm{Ga}\right]$ was 60 . Layers were typically $2 \mu \mathrm{m}$ thick with an erbium concentration of $\sim 10^{17} \mathrm{~cm}^{-3}$, as determined by secondary-ion-mass spectroscopy (SIMS) analysis. The samples were also found to contain oxygen at an amount comparable to the erbium concentration. Growth details are reported elsewhere. ${ }^{3}$ 


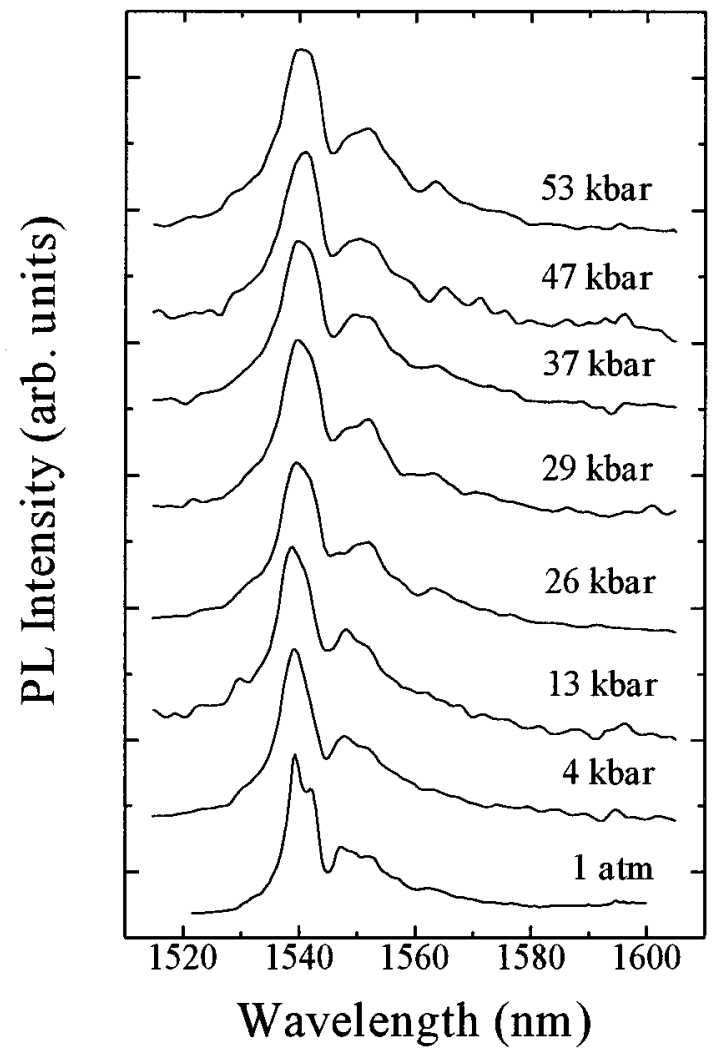

FIG. 1. PL spectra of the ${ }^{4} I_{13 / 2} \rightarrow{ }^{4} I_{15 / 2} \mathrm{Er}^{3+}$ emission in GaAs:Er as a function of pressure at $\sim 45 \mathrm{~K}$. For the spectra other than at $1 \mathrm{~atm}$, the very small DAC sample size results in weaker signals, causing a lower resolution and signal-to-noise ratio.

In the photoluminescence (PL) measurements, samples were cooled in a variable temperature cryostat. Excitation was performed with the $514.5 \mathrm{~nm}$ line of a chopped argon ion laser at an intensity of approximately $13 \mathrm{~W} / \mathrm{cm}^{2}$. Luminescence was collected and dispersed with a one-meter monochromator. In measuring the PL spectra, detection was with a liquid-nitrogen cooled $\mathrm{Ge}$ detector using standard lock-in amplifier techniques. All spectra were corrected for instrumental response. For lifetime measurements, a faster Ge detector with a response time of $\sim 180 \mu$ s was used, and the signal was analyzed with a digital oscilloscope.

Hydrostatic pressure experiments were performed using a diamond anvil cell (DAC) with a 4:1 ethanol:methanol mixture as the pressure-transmitting fluid. Samples to be loaded in the cell were thinned and cleaved to a size approximately $60-70 \mu \mathrm{m}$ thick and $150 \mu \mathrm{m}$ wide. Either ruby ${ }^{4}$ or Tm:YAG ${ }^{5}$ was used as the pressure calibrant.

\section{RESULTS AND DISCUSSION}

\section{A. Spectral characteristics}

The samples used in this study exhibited the characteristic ${ }^{4} I_{13 / 2} \rightarrow{ }^{4} I_{15 / 2} \mathrm{Er}^{3+}$ emission at $1.54 \mu \mathrm{m}$, as well as very weak band edge luminescence. The PL spectra of the ${ }^{4} I_{13 / 2} \rightarrow{ }^{4} I_{15 / 2} \mathrm{Er}^{3+}$ emission at $45 \mathrm{~K}$ and pressures up to 53 kbar are shown in Fig. 1. Additionally, PL spectra at ambient pressure and temperatures ranging from 13 to $300 \mathrm{~K}$ are

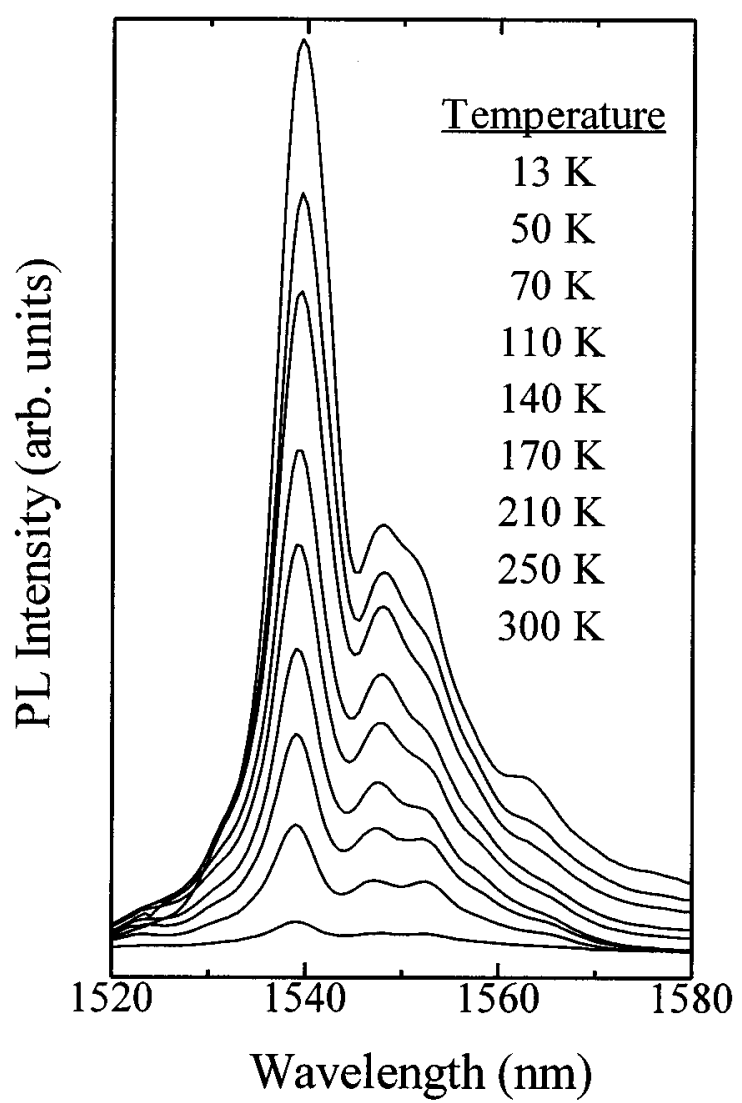

FIG. 2. PL spectra of the ${ }^{4} I_{13 / 2} \rightarrow{ }^{4} I_{15 / 2} \mathrm{Er}^{3+}$ emission in GaAs:Er as a function of temperature at ambient pressure.

shown in Fig. 2. The emission consisted of a main peak at $1539.5 \mathrm{~nm}$ and a group of two overlapping peaks at approximately 1548 and $1552 \mathrm{~nm}$. Over this temperature range, there were no drastic changes in the PL spectrum. As the temperature was increased from 13 to $300 \mathrm{~K}$, the main peak shifted to higher energy by only $5 \AA$. No shift of the main peak was observed as the pressure was increased, within the resolution limit of the pressure experiments. These observations are consistent with the filled outer $5 s$ and $5 p$ electron shells effectively screening the intra- $4 f$ transitions from any changes in the host material.

However, some subtle changes in the emission structure were apparent. The intensity of the $1552 \mathrm{~nm}$ peak decreased with temperature at a rate slower than the $1548 \mathrm{~nm}$ peak. As a results, these two peaks could be resolved at temperatures above $200 \mathrm{~K}$. Furthermore, as the pressure was increased, the $1552 \mathrm{~nm}$ peak grew in intensity relative to the $1548 \mathrm{~nm}$ peak. Finally, it appears that an unresolved shoulder on the low energy side of the main $1540 \mathrm{~nm}$ peak became more significant with increasing pressure.

One interpretation of these results is that many different $\mathrm{Er}^{3+}$ centers in slightly different environments are present. The Stark splitting of the ${ }^{4} I_{15 / 2}$ manifold would be roughly the same for each center, with three of these Stark split levels giving the major peaks at 1540, 1548, and $1552 \mathrm{~nm}$. The slight environmental differences would lead to inhomogeneous broadening of these peaks. In support of this idea, the main peak width was approximately $40 \mathrm{~cm}^{-1}$. This is much 


\section{Aluminum Mole Percent}

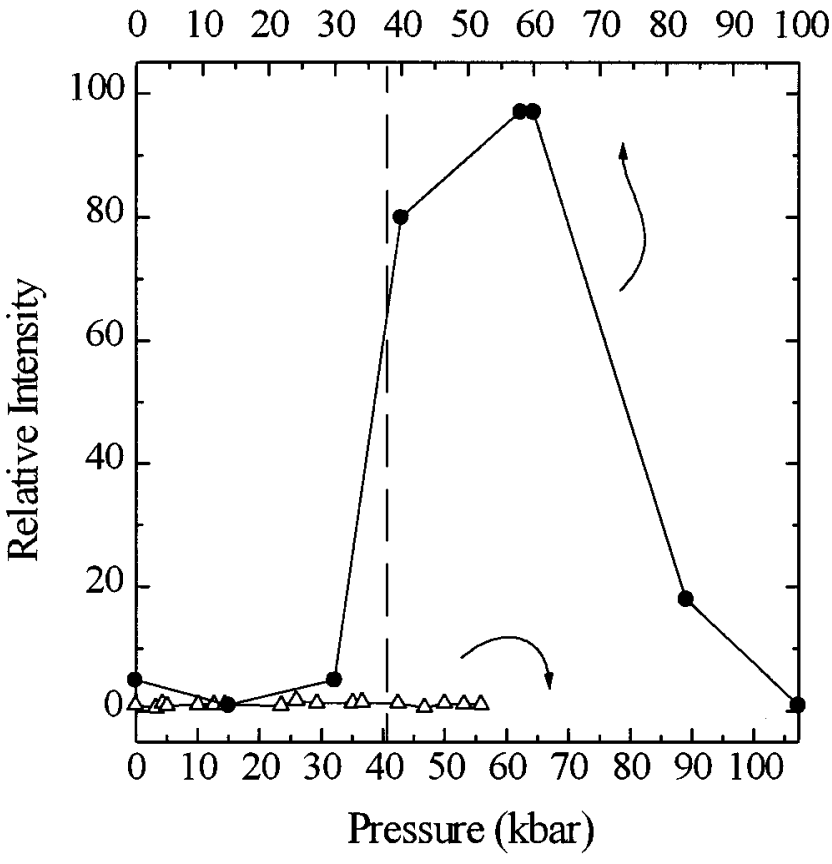

FIG. 3. $\mathrm{Er}^{3+} 1.54 \mu \mathrm{m}$ relative emission intensity as a function of pressure in GaAs, and aluminum content in $\mathrm{Al}_{x} \mathrm{Ga}_{1-x}$ As. Both $x$ axes are scaled to the $\Gamma-X$ crossover, marked by the dotted line. The pressure dependence data (triangles) are at $45 \mathrm{~K}$ with $\mathrm{cw} 514.5 \mathrm{~nm}$ excitation. The aluminum dependence data (circles) are at $77 \mathrm{~K}$ with pulsed $514.5 \mathrm{~nm}$ excitation, although similar results were observed with $\mathrm{cw}$ excitation. The $\mathrm{Al}$ dependence data are from Ref. 9.

broader than emission lines known to come from a single, well-defined center, such as the $\operatorname{Er}_{\mathrm{Ga}}\left(\mathrm{O}_{\mathrm{As}}\right)_{2}$ center in GaAs:Er,O which has a main peak width of only 0.1 $\mathrm{cm}^{-1}{ }^{6,7}$ Although SIMS analysis showed that the sample used in this work does contain oxygen, it was grown at conditions (high growth temperature and V/III ratio) where the $\mathrm{Er}_{\mathrm{Ga}}-\left(\mathrm{O}_{\mathrm{As}}\right)_{2}$ center is not predominant. ${ }^{6,8}$ As a result, many slightly different centers may have contributed to the relatively broad emission. The subtle changes in the emission spectra are due to a combination of two factors. First, for a given center, the transition probabilities between different Stark split levels many have somewhat different temperature and pressure dependencies. Second, the temperature and pressure dependencies of these transition probabilities would also be expected to vary slightly between the different centers.

\section{B. Effects on the low temperature intensity}

At a low temperature, no significant change in the 1.54 $\mu \mathrm{m}$ emission intensity was observed as pressure shifted the $\Gamma$ conduction band edge of GaAs up to and beyond the $\Gamma-X$ crossover at $\sim 41 \mathrm{kbar}$. This contrasts with the behavior observed by Zhang et al. in their studies of erbium doped $\mathrm{Al}_{x} \mathrm{Ga}_{1-x}$ As alloys grown by molecular beam epitaxy. ${ }^{9}$ As the aluminum content was increased, a very strong increase in intensity was observed near the $\Gamma-X$ crossover at $x$ $\sim 0.4$. This type of intensity enhancement has been observed by other researchers as well. ${ }^{10}$ The results are compared in Fig. 3.

Zhang et al. give three possible explanations for the behavior they observed. First, the presence of aluminum somehow enhances the optical activation of erbium centers. Second, the opening of the band gap allows alignment of the conduction bands with upper energy levels of $\mathrm{Er}^{3+}$ such as the ${ }^{4} F_{9 / 2}$ and ${ }^{4} S_{3 / 2}$ manifolds, enhancing excitation efficiency. Third, the $\mathrm{Er}^{3+}$ excitation efficiency increases beyond the $\Gamma-X$ crossover at $x \sim 0.4$ due to the increase in minority carrier lifetime. When the host material has a direct gap, excess carriers have a very short lifetime due to efficient $\Gamma$ band-related recombination, both radiative and nonradiative. ${ }^{11}$ Because the $\mathrm{Er}^{3+}$ luminescence decay is many orders of magnitude slower than band edge recombination, the $\mathrm{Er}^{3+}$ centers cannot effectively compete for the carriers needed for excitation. On the other hand, when the host material has an indirect gap, excess carriers are much longer lived, allowing them a greater probability to excite the $\mathrm{Er}^{3+}$ centers.

The application of hydrostatic pressure is a unique tool to test these proposed explanations. There is a qualitative correspondence between the effects of applying pressure and alloying with aluminum on the band structure. The $\Gamma$ conduction band edge shifts up in energy at a comparable rate per kbar pressure in GaAs or percent aluminum content in $\mathrm{Al}_{x} \mathrm{Ga}_{1-x} \mathrm{As}$. Furthermore, the direct to indirect transition ( $\Gamma-X$ crossover) occurs at $x \sim 40 \%$ in $\mathrm{Al}_{x} \mathrm{Ga}_{1-x} \mathrm{As}$, and $P$ $\sim 41 \mathrm{kbar}$ in GaAs. It should be noted that this rough equivalence between percent aluminum content and pressure in kbar breaks down far above the crossover point because the indirect band gap continues to increase with aluminum content whereas it slowly decreases with pressure. ${ }^{12}$ Nevertheless, pressure allows a qualitative comparison between GaAs and $\mathrm{Al}_{x} \mathrm{Ga}_{1-x} \mathrm{As}$ systems up to and slightly beyond the direct-indirect transition.

The pressure results in Fig. 3 appear to rule out the last two explanations offered by Zhang et al. Applying pressure on GaAs:Er simulates the same behavior in the band structure, including possible alignment of the conduction bands with upper energy $\mathrm{Er}^{3+}$ levels and the increase in excess carrier lifetime beyond the $\Gamma-X$ crossover. However, no significant increase in emission intensity was observed.

Zhang et al.'s first explanation that the presence of $\mathrm{Al}$ somehow enhances the optical activation of $\mathrm{Er}^{3+}$ centers is a chemical effect which cannot be excluded by the pressure results. Thus, the intensity enhancement seen in $\mathrm{Al}_{x} \mathrm{Ga}_{1-x} \mathrm{As}$ :Er is most likely due to an alloying effect related to the presence of $\mathrm{Al}$ rather than a consequence of the band gap or indirect behavior of the host. We speculate that the increase in erbium intensity is due to incorporation of an impurity such as oxygen along with Al. Aluminum alloying in both MOCVD and MBE $\mathrm{Al}_{x} \mathrm{Ga}_{1-x}$ As is known to bring residual oxygen impurities into the host material in high concentrations. ${ }^{13,14}$ Oxygen codoping is known to enhance the $\mathrm{Er}^{3+}$ emission in GaAs and $\mathrm{Al}_{x} \mathrm{Ga}_{1-x}$ As. ${ }^{8,15}$ It should be reiterated that the GaAs:Er sample used in this work also contains oxygen, but the amount of oxygen will obviously 


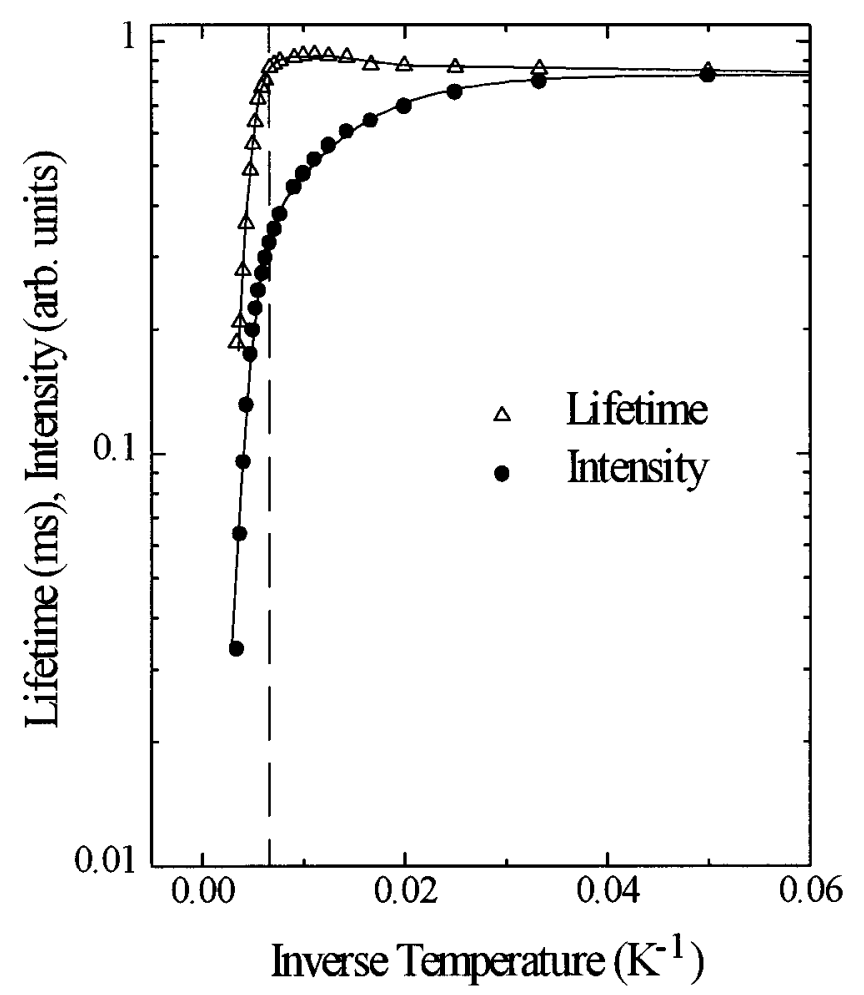

FIG. 4. Temperature dependence of the $1.54 \mu \mathrm{m} \mathrm{Er}{ }^{3+}$ emission intensity and lifetime at ambient pressure. The dotted line, located at approximately $150 \mathrm{~K}$, indicates the two regions of different quenching behavior. For clarity, 95\% confidence intervals have been omitted from the plot. These intervals are approximately $\pm 0.003 \mathrm{~ms}$ for the intensity, $\pm 0.006 \mathrm{~ms}$ for the lifetime at low temperatures, and $\pm 0.018 \mathrm{~ms}$ for the lifetime at high temperatures. The curves are fitted results to Eq. (1).

remain constant in the pressure experiments. Thus, the base level of intensity enhancement that may be occurring in this sample is present at all pressures, whereas oxygen incorporation and enhancement can continue to increase with aluminum alloying.

\section{Thermal quenching properties}

As is evident in Fig. 2, the intensity of the $1.54 \mu \mathrm{m}$ emission decreased rapidly with increasing temperature. Figure 4 shows this thermal quenching more explicitly, for both the peak emission intensity and lifetime at ambient pressure. The temperature dependence of the integrated emission intensity is practically identical to that of the peak emission intensity. In the time-resolved PL measurements, the luminescence was found to follow a single exponential decay, from which the lifetime was determined. The fact that the decay is single exponential indicates that the different $\mathrm{Er}^{3+}$ centers contributing to the relatively broad emission peak have similar lifetimes. Two distinct regions are apparent in Fig. 4, as indicated by the line at approximately $150 \mathrm{~K}$. At low temperatures, the intensity decreased with increasing temperature, whereas the lifetime did not. In fact, there is a slight lifetime increase from $0.84 \mathrm{~ms}$ at $12 \mathrm{~K}$ to $0.93 \mathrm{~ms}$ at 90 $\mathrm{K}$. This behavior suggests a low activation energy quenching process which prevents $\mathrm{Er}^{3+}$ excitation but does not affect the $\mathrm{Er}^{3+}$ ion once it is excited. As a result, the intensity is

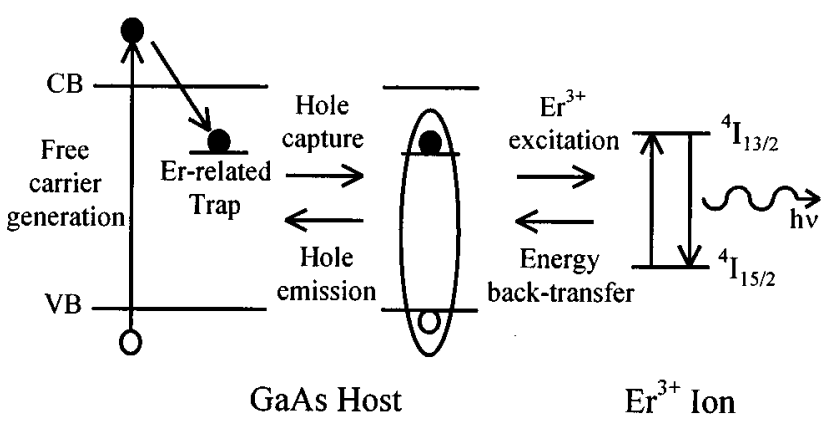

FIG. 5. Model for energy transfer between the semiconductor host and the rare-earth ion, showing the processes of excitation, energy back transfer, and hole emission. An electron trap is assumed for the rare-earth center.

reduced, but the measured lifetime is largely unchanged. At temperatures above $\sim 150 \mathrm{~K}$, the intensity continued to quench, and the lifetime also began to decrease rapidly with increasing temperature. This region indicates a higher activation energy process which depopulates the excited $\mathrm{Er}^{3+}$ ion, quenching both the intensity and lifetime.

The data can be fit to a one- or two-process quenching model of the form: ${ }^{16,17}$

$$
X=\frac{X_{0}}{1+A_{1} \exp \left(-\frac{E_{1}}{k T}\right)+A_{2} \exp \left(-\frac{E_{2}}{k T}\right)},
$$

where $X$ is either the intensity, $I$, or the lifetime, $\tau$. The temperature dependence of the $1.54 \mu \mathrm{m}$ intensity at ambient pressure can be fit excellently with the two-process model. The fitted activation energies are $E_{1}=13.4 \pm 0.3$ and $E_{2}$ $=115.8 \pm 3.4 \mathrm{meV}$, where the indicated errors are $95 \%$ confidence intervals. Values similar to these have been previously reported. ${ }^{18}$ The temperature dependence of the lifetime was fit very well with only a one-process model, yielding an activation energy of $E=113.1 \pm 10.0 \mathrm{meV}$. This value closely matches the second activation energy determined from the intensity quenching, suggesting that they may be related to the same process.

In order to consider possible quenching models, it is necessary to first discuss the excitation mechanism. The most widely accepted model for energy transfer between the semiconductor host and the rare-earth ion was first discussed for II-VI compounds ${ }^{19,20}$ and InP:Yb ${ }^{21,22}$ followed by a similar model for GaAs:Er. ${ }^{18,23}$ In this model, the rare-earth ion introduces either an electron trap below the conduction band or a hole trap above the valence band. After free carriers are created in the host material by minority-carrier injection or absorption of above-band gap light, either an electron or hole is trapped at the rare-earth center. An opposite carrier is then attracted to the charged center through its long-range Coulombic potential, forming a bound exciton localized on the rare-earth ion. The exciton recombines and transfers its energy to the $4 f$ shell of the rare-earth ion. Finally, the excited rare-earth ion can decay to its ground state, emitting a photon. This process is shown in Fig. 5, assuming that $\mathrm{Er}^{3+}$ introduces an electron trap. 


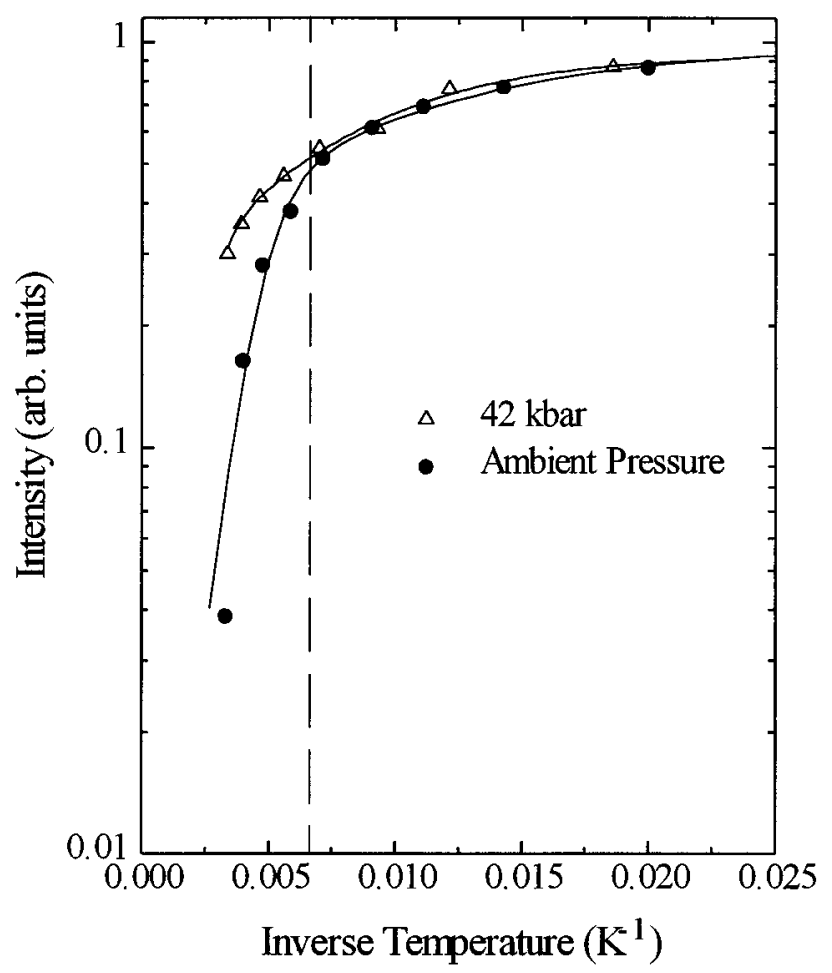

FIG. 6. The effect of pressure on the thermal quenching of the $1.54 \mu \mathrm{m}$ $\mathrm{Er}^{3+}$ emission intensity. The dotted line at approximately $150 \mathrm{~K}$ indicates the two regions of interest. On this normalized scale, $95 \%$ confidence intervals are approximately \pm 0.003 for the ambient pressure data and \pm 0.03 for the high pressure data.

The $13 \mathrm{meV}$ thermal quenching process, active at low temperatures, reduced the intensity but not the lifetime. One possible assignment is the thermal dissociation of the bound exciton before it can recombine to excite the $\mathrm{Er}^{3+}$ ion. If $\mathrm{Er}^{3+}$ introduces an electron trap, this process corresponds to hole emission, leaving the electron trapped on the Er center. The coulombic electron-hole binding energy is expected to be on the order of this activation energy. ${ }^{12}$ This mechanism does not affect the $\mathrm{Er}^{3+}$ ion once it is excited, but rather prevents initial $\mathrm{Er}^{3+}$ excitation. As a result, the intensity is quenched, but the lifetime is unaffected, as was observed in the low temperature region. This type of process has been previously proposed for InP: $\mathrm{Yb}^{22}$ and GaAs:Er. ${ }^{18}$

The $\sim 115 \mathrm{meV}$ process, active at higher temperatures, quenched both the intensity and lifetime. This mechanism is most likely "energy back transfer" as proposed by Thonke et al. and Taguchi et al. ${ }^{22,24}$ The excited $\mathrm{Er}^{3+}$ ion decays and transfers its energy to reform the bound exciton, rather than release a photon. As shown in Fig. 5, this is simply the reverse of the excitation mechanism. In this mechanism, the excited $\mathrm{Er}^{3+}$ ion is deactivated, so both the intensity and lifetime will be quenched with the same activation energy, as was observed at temperatures above $\sim 150 \mathrm{~K}$.

The temperature dependence of the $\mathrm{Er}^{3+}$ luminescence was also examined at high pressure to gain further insight into the assignment of these quenching mechanisms. Figure 6 compares the temperature dependence of the emission intensity at ambient pressure and $42 \mathrm{kbar}$. At ambient pressure, the intensity was quenched by a factor of $\sim 25$ as the tem- perature was increased from 12 to $296 \mathrm{~K}$. However, at 42 kbar, the thermal quenching was reduced by nearly one order of magnitude at room temperature. Again, two distinct regions are apparent. The quenching process dominant at low temperatures was largely unaffected by the increase in pressure, whereas the process dominant at higher temperatures was significantly reduced.

A satisfactory fit of the intensity data at $42 \mathrm{kbar}$ requires two processes in the quenching model, but the first process dominates over the minor, but non-negligible, second process. The dominant process has an activation energy of $E_{1}$ $=10.4 \pm 3.0 \mathrm{meV}$. The values for $E_{1}$ at ambient pressure and 42 kbar are comparable, again demonstrating that the low activation energy process is fairly independent of pressure. The second process is so weak that $E_{2}$ is very uncertain. By varying the prefactor $A_{2}$ over a large range, satisfactory fits are obtained for values extending from 40 to $350 \mathrm{meV}$. However, the prefactor is related to the degeneracies of the levels involved in the quenching process, as well as the matrix element of the energy transfer transition. ${ }^{16,17,25}$ These quantities should not have a strong dependence upon pressure. ${ }^{25}$ Figure 3 supports this fact, in that the low temperature intensity is nearly constant at pressures up to 56 kbar. Thus, to determine a more realistic range for $E_{2}$, we might assume $A_{2}$ has the same value at $42 \mathrm{kbar}$ as at ambient pressure. With this assumption, $E_{2}$ can now be estimated to lie within the narrowed range of $140-300 \mathrm{meV}$ at $42 \mathrm{kbar}$. The pressure shift rate of the activation energy is then estimated to be between +0.6 and $5 \mathrm{meV} / \mathrm{kbar}$. At $42 \mathrm{kbar}$, the higher activation energy process has been considerably reduced, due to an increase in the activation energy.

Figure 7 compares the temperature dependence of the emission lifetime at ambient pressure and $42 \mathrm{kbar}$. There was a small increase in the low temperature lifetime at $42 \mathrm{kbar}$. Furthermore, at high pressure, the lifetime appeared not to quench at temperatures up to and possibly beyond $230 \mathrm{~K}$, whereas the ambient pressure lifetime decreased rapidly above approximately $150 \mathrm{~K}$. However, we should emphasize that the uncertainty of the $42 \mathrm{kbar}$ lifetimes is rather large at these higher temperatures. In the high-pressure lifetime measurements, the combined factors of the extremely small sample size in the diamond anvil cell, the need for a fast Ge detector, and the thermal quenching of the intensity all lead to very low signal levels at higher temperatures. As a result, the uncertainty of the lifetime increases. Despite the uncertainty, this data again suggests that the higher activation energy process was significantly diminished at high pressure.

The temperature dependence of the $\mathrm{Er}^{3+}$ emission intensity and lifetime at high pressure supports the assignments previously made for the two quenching mechanisms. First, the lower activation energy process, proposed to be thermal dissociation of the bound exciton, was largely unchanged at high pressure. This observation is consistent with the assignment, since the electron-hole coulombic binding energy is not expected to change much with pressure. ${ }^{25}$

Next, the pressure-induced reduction of the higher activation energy process, characterized by an increase in the activation energy, is consistent with the assignment of the process as energy back transfer. This activation energy is 


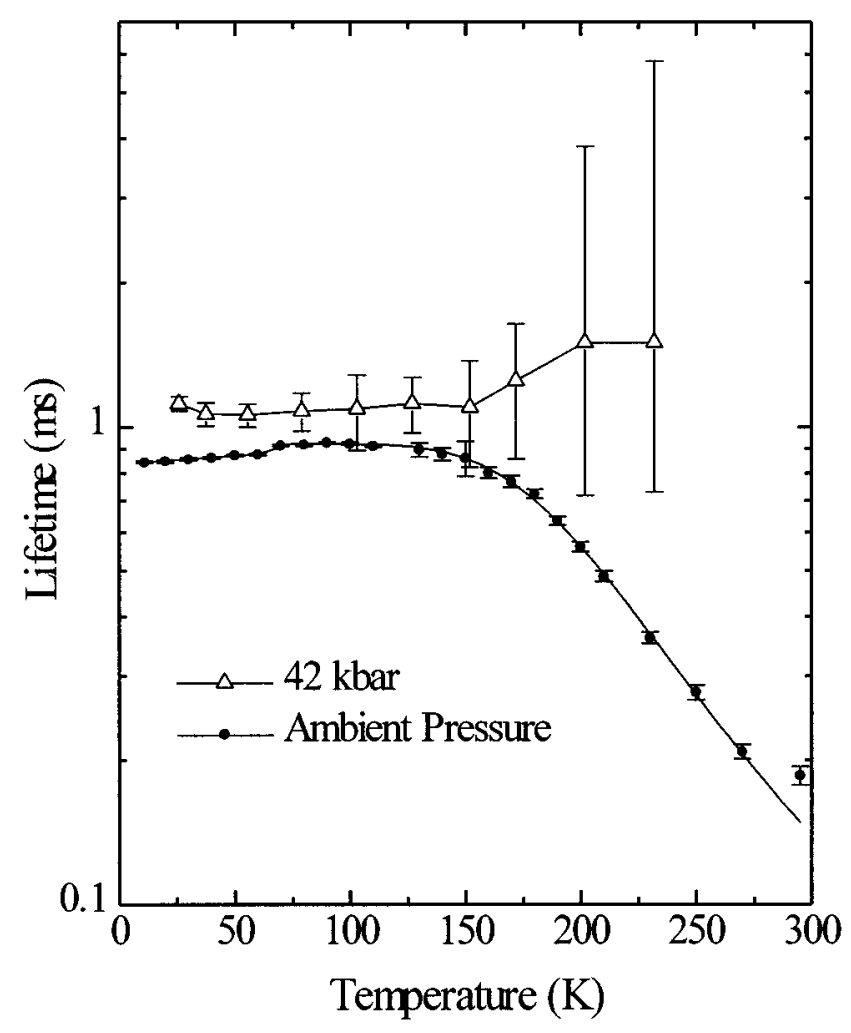

FIG. 7. The effect of pressure on the thermal quenching of the $1.54 \mu \mathrm{m}$ $\mathrm{Er}^{3+}$ emission lifetime. The error bars indicate $95 \%$ confidence intervals. In the high-pressure lifetime measurements, the very low signal levels lead to increased uncertainty at higher temperatures.

equal to the bound exciton recombination energy minus the $\mathrm{Er}^{3+}{ }^{4} I_{13 / 2} \rightarrow{ }^{4} I_{15 / 2}$ transition energy. The $\mathrm{Er}^{3+}$ transition energy is independent of pressure, as shown in Fig. 1. Thus, the experimental results indicate that the bound exciton recombination energy is increasing with pressure in this model. The rate of increase is difficult to specify with precision, but is likely to lie between 0.6 and $5 \mathrm{meV} / \mathrm{kbar}$. If we assume that $\mathrm{Er}^{3+}$ introduces an electron trap, the increasing bound exciton recombination energy implies that the Er-related trap shifts up in energy relative to the valence band at a rate between 0.6 and $5 \mathrm{meV} / \mathrm{kbar}$. For comparison, the $\Gamma$ conduction band edge increases in energy relative to the valence band at a rate of $10.7 \mathrm{meV} / \mathrm{kbar}^{26}{ }^{26}$ Figure 8 shows the effect of this pressure shift on the energy back-transfer process. A similar model for the pressure shift of the rare-earth related trap has been proposed for InP:Yb ${ }^{25,27}$ and GaAs:Er,O. ${ }^{28}$

This model is consistent with other experimental and theoretical predictions for the shift rate of the Er-related level. Takarabe et al. report a shift rate of $+3.6 \mathrm{meV} / \mathrm{kbar}$ for the Er-related trap in GaAs:Er,O. ${ }^{28}$ Next, if we assume that the deep electron mixes states from all bands, a degeneracy-weighted average across the Brillouin zone gives a shift rate of $+2.4 \mathrm{meV} / \mathrm{kbar}$ relative to the valence band. ${ }^{29}$ Finally, if we assume the Er is substitutional on a Ga site, tight-binding calculations predict a shift rate of 1-4 $\mathrm{meV} / \mathrm{kbar}^{30}$ All of these values are consistent with the present study, and support the assignment of the second quenching process as energy back transfer.

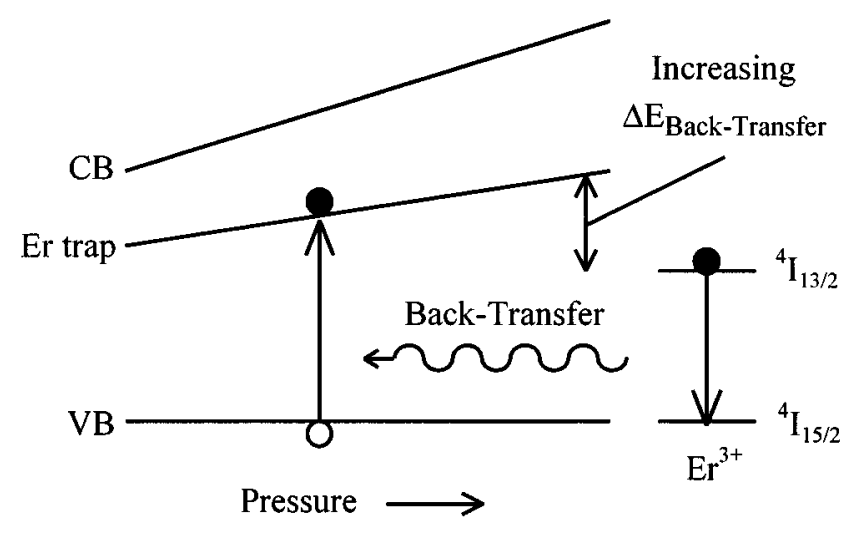

FIG. 8. The effect of pressure on the energy back-transfer quenching mechanism. The $\mathrm{Er}^{3+}$ ground state is arbitrarily aligned with the valence band to allow easy identification of the back-transfer activation energy. The absolute positions of the $\mathrm{Er}^{3+}$ levels relative to the bands are not important; only the difference between the bound exciton recombination energy and the ${ }^{4} I_{13 / 2} \rightarrow{ }^{4} I_{15 / 2}$ transition energy is significant.

Nearly an order of magnitude increase in emission intensity at room temperature was obtained due to the reduction of energy back transfer at 42 kbar. However, despite this gain, the intensity still decreased by a factor of 3 to 4 at room temperature due to the other quenching process, thermal dissociation of the bound exciton. Thus, the fairly low coulombic electron-hole binding energy sets a serious limit on the room temperature intensity. It is well known that thermal quenching of the $\mathrm{Er}^{3+}$ emission is significantly less in wide band gap materials. ${ }^{31,32}$ In fact, the $1.54 \mu \mathrm{m}$ intensity is nearly constant up to $400 \mathrm{~K}$ in $6 \mathrm{H} \mathrm{SiC:Er}\left(E_{G} \approx 3.0 \mathrm{eV}\right){ }^{33}$ One common explanation is that the Er-related trap position varies such that energy back transfer is greatly diminished in wide band gap hosts. However, in view of the present study, the observed independence of the intensity at high temperatures must actually be due to not only a reduction in energy back transfer, but also a reduction of thermal dissociation of the bound exciton. In support of this idea, there is a general trend of increasing free exciton binding energy with band gap energy, as a result of the increasing free carrier effective mass and decreasing dielectric constant. ${ }^{34}$ The coulombic binding energy at a localized rare-earth center may vary from that of a free exciton, but the trend is likely still valid.

\section{SUMMARY}

The characteristics of the ${ }^{4} I_{13 / 2} \rightarrow{ }^{4} I_{15 / 2} \mathrm{Er}^{3+}$ emission of MOCVD GaAs:Er were investigated as a function of temperature and applied hydrostatic pressure. $\mathrm{The}^{\mathrm{Er}^{3+}}$ emission wavelength was largely independent of both pressure and temperature, due to the shielded nature of the intra- $4 f$ transitions. The low temperature emission intensity was also independent of pressure up to and beyond the $\Gamma-X$ crossover, in contrast with the strong intensity increase observed in $\mathrm{Al}_{x} \mathrm{Ga}_{1-x} \mathrm{As}$ :Er alloys. This enhancement is most likely due to a chemical effect related to the presence of $\mathrm{Al}$, such as residual oxygen incorporation, rather than a band structure effect related to the indirect band gap or larger band gap energy. 
The temperature dependence of the $\mathrm{Er}^{3+}$ emission intensity and lifetime at ambient pressure suggests two dominant quenching mechanisms: a low activation energy process which prevents $\mathrm{Er}^{3+}$ excitation, and a higher activation energy process which deactivates the excited $\mathrm{Er}^{3+}$ ion. At high pressure, the low activation energy process was largely unaffected, whereas the higher activation energy process was significantly reduced. These processes are proposed to be thermal dissociation of the Er-bound exciton, and energy back transfer, respectively. Although these mechanisms have been proposed previously by several groups, the direct comparison of lifetime and intensity quenching in this study at both ambient and high pressure allows a more definitive assignment of the dominant quenching mechanisms. Finally, we have presented a model in which the Er-related electron trap shifts up in energy at higher pressure, increasing the activation energy to back transfer, but not affecting thermal dissociation of the bound exciton through hole emission.

\section{ACKNOWLEDGMENTS}

We gratefully acknowledge financial support under grants from the National Science Foundation and Army Research Office, as well as a National Science Foundation Graduate Research Fellowship.

${ }^{1}$ Rare Earth Doped Semiconductors, Materials Research Society Symposium Proceedings 1993, edited by G. S. Pomrenke, P. B. Klein, and D. W. Langer (Materials Research Society, Pittsburgh, PA, 1993), Vol. 301.

${ }^{2}$ Rare Earth Doped Semiconductors II, Materials Research Society Symposium Proceedings 1996, edited by S. Coffa, A. Polman, and R. N. Schwartz (Materials Research Society, Pittsburgh, PA, 1996), Vol. 422.

${ }^{3}$ J. M. Redwing, D. C. Gordon, B. A. Vaartstra, S. S. Lau, and T. F. Kuech, J. Appl. Phys. 76, 1585 (1994).

${ }^{4}$ R. A. Forman, G. J. Piermarini, J. D. Barnett, and S. Block, Science 176, 284 (1972).

${ }^{5}$ P. R. Wamsley and K. L. Bray, J. Lumin. 60\&61, 188 (1994).

${ }^{6}$ H. Nakagome, K. Uwai, and K. Takahei, Appl. Phys. Lett. 53, 1726 (1988).

${ }^{7}$ K. Takahei, A. Taguchi, Y. Horikoshi, and J. Nakata, J. Appl. Phys. 76, 4332 (1994).
${ }^{8}$ K. Takahei and A. Taguchi, J. Appl. Phys. 74, 1979 (1993).

${ }^{9}$ T. Zhang, J. Sun, N. V. Edwards, D. E. Moxey, R. M. Kolbas, and P. J. Caldwell, Mater. Res. Soc. Symp. Proc. 301, 257 (1993).

${ }^{10}$ P. Galtier, T. Benyattou, J. P. Pochelle, M. N. Charasse, G. Guillot, and J. P. Hirtz, Inst. Phys. Conf. Ser. 106, 327 (1990).

${ }^{11}$ S. Gupta, S. Sethi, and P. K. Bhattacharya, Appl. Phys. Lett. 62, 1128 (1993).

${ }^{12}$ L. Pavesi and M. Guzzi, J. Appl. Phys. 75, 4779 (1994).

${ }^{13}$ T. F. Kuech, R. Potemski, F. Cardone, and G. Scilla, J. Electron. Mater. 21, 341 (1992).

${ }^{14}$ K. Akimoto, M. Kamada, K. Taira, M. Arai, and N. Watanabe, J. Appl. Phys. 59, 2833 (1986).

${ }^{15}$ J. E. Colon, D. W. Elsaesser, Y. K. Yeo, R. L. Hengehold, and G. S. Pomrenke, Appl. Phys. Lett. 63, 216 (1993).

${ }^{16}$ D. Bimburg, M. Sondergeld, and E. Grobe, Phys. Rev. B 4, 3451 (1971).

${ }^{17}$ F. E. Williams and H. Eyring, J. Chem. Phys. 15, 289 (1947).

${ }^{18}$ J. E. Colon, D. W. Elsaesser, Y. K. Yeo, R. L. Hengehold, and G. S. Pomrenke, Mater. Sci. Forum 83-87, 671 (1992).

${ }^{19}$ R. Boyn, Phys. Status Solidi B 148, 11 (1988).

${ }^{20}$ H. Przybylinska, K. Swiatek, A. Stapor, A. Suchocki, and M. Godlewski, Phys. Rev. B 40, 1748 (1989).

${ }^{21}$ K. Takahei, A. Taguchi, H. Nakagome, K. Uwai, and P. S. Whitney, J. Appl. Phys. 66, 4941 (1989).

${ }^{22}$ K. Thonke, K. Pressel, G. Bohnert, A. Stapor, J. Weber, M. Moser, A. Molassioti, A. Hangleiter, and F. Scholz, Semicond. Sci. Technol. 5, 1124 (1990)

${ }^{23}$ T. Benyattou, D. Seghier, G. Guillot, R. Moncorge, P. Galtier, and M. N. Charasse, Mater. Res. Soc. Symp. Proc. 163, 69 (1990).

${ }^{24}$ A. Taguchi, H. Nakagome, and K. Takahei, Appl. Phys. Lett. 60, 965 (1992).

${ }^{25}$ A. Taguchi, K. Takahei, and Y. Horikoshi, J. Appl. Phys. 76, 7288 (1994).

${ }^{26}$ D. J. Wolford and J. A. Bradley, Solid State Commun. 53, 1069 (1985).

${ }^{27}$ K. Takarabe, S. Minomura, A. Taguchi, and K. Takahei, Mater. Sci. Forum 143-147, 731 (1994).

${ }^{28}$ K. Takarabe, T. Mizushima, S. Minomura, A. Taguchi, and K. Takahei, Mater. Sci. Forum 196-201, 645 (1995).

${ }^{29}$ D. J. Chadi and K. J. Chang, Phys. Rev. Lett. 61, 873 (1988).

${ }^{30}$ S. Y. Ren, J. D. Dow, and D. J. Wohlford, Phys. Rev. B 25, 7661 (1982).

${ }^{31}$ P. N. Favennec, H. L'Haridon, M. Salvi, D. Moutonnet, and Y. Le Guillou, Electron. Lett. 25, 718 (1989).

${ }^{32}$ A. J. Neuhalfen and B. W. Wessels, Appl. Phys. Lett. 60, 2657 (1992).

${ }^{33}$ M. Yoganathan, W. J. Choyke, R. P. Devaty, G. Pensl, and J. A. Edmond, Mater. Res. Soc. Symp. Proc. 422, 339 (1996).

${ }^{34}$ C. F. Klingshirn, Semiconductor Optics (Springer, New York, 1995), p. 166. 